\title{
Sonography of Uterine Abnormalities in Postpartum and Postabortion Patients
}

\section{A Potential Pitfall of Interpretation}

\author{
Stephanie L. Rufener, MD, Saroja Adusumilli, MD, + \\ William J. Weadock, MD, Elaine Caoili, MD
}

\begin{abstract}
Objective. The interpretation of postpartum and postabortion uterine abnormalities on sonography can be challenging. The purpose of this study was to identify misleading imaging features that lead to inclusion of a uterine arteriovenous malformation (AVM) in the differential diagnosis of a uterine abnormality because consideration of this diagnosis can potentially alter patient treatment. Methods. The sonographic examinations of 29 pathologically proven cases of uterine abnormalities in postpartum and postabortion patients were retrospectively reviewed. Two radiologists independently evaluated several features: the presence of a uterine mass, myometrial involvement, and the presence of an associated vascular abnormality. Percent agreement and the relationship between imaging features and inclusion of a uterine AVM in the differential diagnosis were determined. Results. Interobserver agreement was as follows: the presence of a uterine mass, $90 \%$; myometrial involvement, $83 \%$; the presence of an associated vascular abnormality, $72 \%$; and inclusion of a uterine AVM in the differential diagnosis, $86 \%$. Myometrial involvement showed a statistically significant relationship to inclusion of a uterine AVM in the differential diagnosis $(P<.05)$. Final pathologic diagnoses included retained products of conception (RPOC) $(n=26)$, an endometrial polyp $(n=1)$, chronic endometritis $(n=1)$, and an exogenous progestational effect $(n=1)$. No uterine AVMs were found. Conclusions. Despite high interobserver agreement in characterizing uterine abnormalities on sonography, readers still include uterine AVMs in the differential diagnosis of uterine masses that are ultimately proven to be RPOC. A myometrial location of a uterine mass is a particularly misleading imaging feature of RPOC. Key words: postabortion; postpartum; retained products of conception; sonography; uterine arteriovenous malformation.
\end{abstract}

\begin{abstract}
Abbreviations
AVM, arteriovenous malformation; D\&C, dilation and

curettage; RPOC, retained products of conception

Received November 5, 2007, from the Department of Radiology, University of Michigan Health System, 1500 E Medical Center Dr, Ann Arbor, Michigan USA. Revision requested November 19, 2007. Revised manuscript accepted for publication December 4, 2007.

We thank Matthew Schipper, PhD, for assistance in statistical calculations.

tSaroja Adusumilli, MD, was tragically killed in an automobile collision on March 3, 2007. Her selfless dedication to patient care, resident education, and the radiology community continue to make her a role model for all who knew her and for those who encounter her work.

Address correspondence to William J. Weadock, MD, Department of Radiology, University of Michigan Hospitals, 1500 E Medical Center Dr, UHB1D407, Box 0030, Ann Arbor, MI 48109-0030 USA.

E-mail:weadock@med.umich.edu
\end{abstract}

nterpretation of postpartum and postabortion uterine abnormalities on sonography can be challenging. The primary reason to use sonography in this setting is to evaluate for retained products of conception (RPOC), a known complication that can lead to prolonged hemorrhage and endometritis. Clinical diagnosis of RPOC is difficult because symptoms are nonspecific and can be similar to symptoms occurring after normal delivery and spontaneous and induced abortions. The sonographic features of RPOC are also often nonspecific because blood clots and RPOC can have similar sonographic appearances. ${ }^{1}$ 
The differential diagnosis of postpartum and postabortion vaginal bleeding also includes a uterine arteriovenous malformation (AVM). Certain imaging features can influence the sonologist to include entities such as a uterine $\mathrm{AVM}$ in the differential diagnosis, which can affect patient treatment. A study by Huang et $\mathrm{al}^{2}$ looked retrospectively at sonographic features of uterine AVMs in 10 patients. One of their conclusions was that the gray scale and color Doppler features of uterine AVMs may overlap with other causes of arteriovenous shunting such as abnormal placentation with RPOC. ${ }^{2}$

The purpose of this study was to identify misleading imaging features that lead to inclusion of a uterine AVM in the differential diagnosis of a uterine abnormality because consideration of this diagnosis can potentially alter patient treatment.

\section{Materials and Methods}

Institutional Review Board approval was obtained, and acquisition of consent was waived for this retrospective study. The study group was identified by performing a radiologic database search of pelvic sonography reports containing the following key words: abortion, retained products of conception, arteriovenous malformation, and arteriovenous fistula. The final dictated reports were reviewed and cross-referenced with patient medical records to identify a group of postpartum and postabortion patients with abnormal findings on pelvic sonography who had undergone subsequent evacuation of the uterine cavity with surgical specimens sent for pathologic analysis.

\section{Study Population}

The study population included 29 female patients ranging in age from 17 to 46 years. Twenty-one patients were of postabortion status. The approximate mean gestational age at the time of abortion was 11 weeks, with a range of 7 to 20 weeks. In 4 of the 21 postabortion patients, the mean gestational age at the time of abortion was not known with certainty. Eight patients were of postpartum status. Five of the 8 postpartum patients had term vaginal deliveries. Pelvic sonographic examinations were performed between February 2001 and April 2005. The mean time between delivery or abortion and uterine imaging was 29 days, with a range of 1 to 114 days. All of the patients had histories of recent vaginal bleeding and underwent evacuation of the uterine cavity with surgical specimens sent for pathologic analysis.

\section{Sonographic Interpretations}

Transabdominal and transvaginal imaging was performed with 1 of 3 sonography machines: Acuson Sequoia (Siemens Medical Solutions USA, Inc, Malvern, PA), HDI 500 (Philips Medical Systems, Bothell, WA), and LOGIQ 9 (GE Healthcare, Milwaukee, WI). Two radiologists, both body imaging subspecialists, independently reviewed the sonographic examinations retrospectively and specifically evaluated the following features: the presence of a uterine mass, myometrial involvement, the presence of an associated vascular abnormality, and whether they would include a uterine AVM in the differential diagnosis. Twenty-six of the studies included both static and cine images and were viewed on a computer monitor. Three of the studies were printed on hard copy film and contained static images only. Color Doppler evaluation of the uterus was available for all patients.

\section{Analysis}

Frequency tables were created to evaluate for percent agreement between the two radiologists. A Fisher exact $\chi^{2}$ test was performed to evaluate the relationship between imaging features and inclusion of a uterine AVM in the differential diagnosis. $P<.05$ was considered significant.

\section{Results}

Radiologists 1 and 2 both found that a uterine mass was present in most cases (28/29 and 27/29 cases, respectively). Radiologists 1 and 2 both characterized the dominant abnormality as endometrial in location in most cases (28/29 and 23/29 cases). Radiologist 1 found a vascular abnormality to be present in 14 of 29 cases; radiologist 2 found a vascular abnormality in 18 of 29 . Radiologist 1 included a uterine AVM in the differential diagnosis for 1 of 29 cases; radiologist 2 included a uterine AVM for 5 of 29. These results are summarized in Figure 1. 


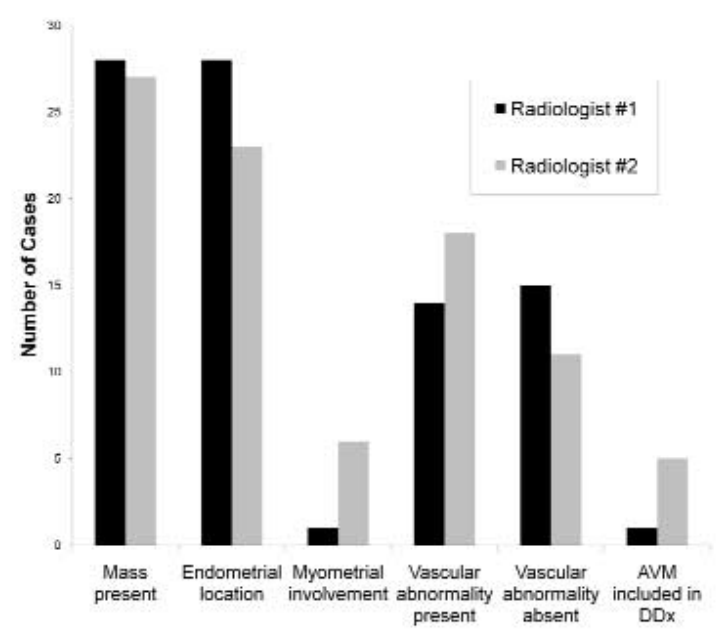

Figure 1. Summary of sonographic findings evaluated independently by two radiologists. DDx indicates differential diagnosis.

Interobserver agreement was as follows: the presence of a uterine mass, $90 \%$; myometrial involvement, 83\%; the presence of an associated vascular abnormality, $72 \%$; and inclusion of a uterine AVM in the differential diagnosis, $86 \%$. Myometrial involvement showed a statistically significant relationship to inclusion of a uterine AVM in the differential diagnosis $(P=.00005$ and .034 for radiologists 1 and 2, respectively). The presence of a uterine mass $(P<.9$ for both radiologists) and the presence of an associated vascular abnormality $(P=.13$ and .48 for radiologists
1 and 2, respectively) showed no statistically significant relationship to inclusion of a uterine AVM in the differential diagnosis.

Final pathologic diagnoses included RPOC ( $n=26)$, an endometrial polyp ( $n=1)$, chronic endometritis $(n=1)$, and exogenous progestational effect $(n=1)$. The most common pathologic diagnosis was RPOC. No uterine AVMs were found. Examples of sonographic findings and radiologic interpretations with corresponding pathologic diagnoses are shown in Figures 2-4.

\section{Discussion}

Determining the etiology of postpartum and postabortion bleeding can be challenging from both a clinical and radiologic perspective. The challenge in sonographic interpretation often lies in trying to identify the presence of RPOC. A 2005 study by Durfee et $\mathrm{al}^{3}$ evaluated the sonographic and color Doppler features of RPOC in 163 patients with clinically suspected RPOC. The investigators found that an endometrial mass was the most sensitive $(79 \%)$ and specific (89\%) sonographic feature for RPOC. However, sonographic findings of RPOC are notoriously nonspecific; for instance, the presence of blood clots or infected or necrotic material can also appear as a heterogeneous mass in the endometrial cavity in the absence of RPOC. ${ }^{4}$ The difficulty of

Figure 2. Transvaginal sonography without (A) and with (B) color Doppler imaging shows findings characterized as a vascular endometrial mass by both radiologists. The final pathologic diagnosis was RPOC.

A

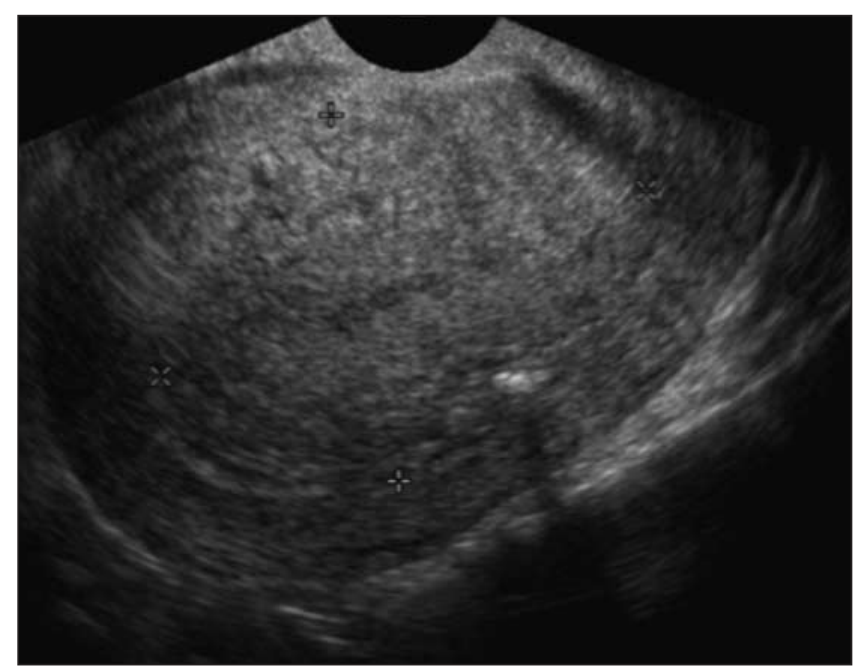

B

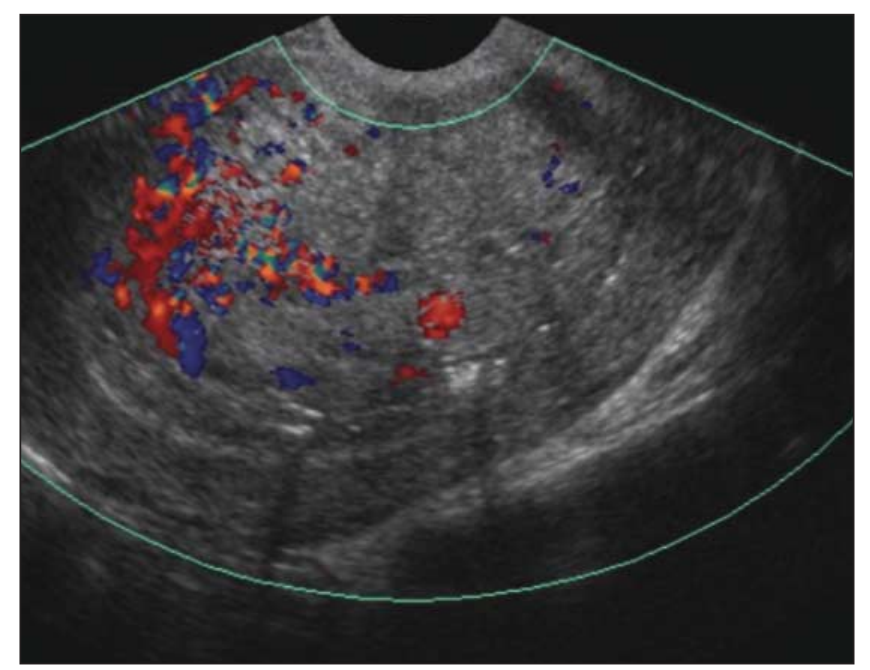




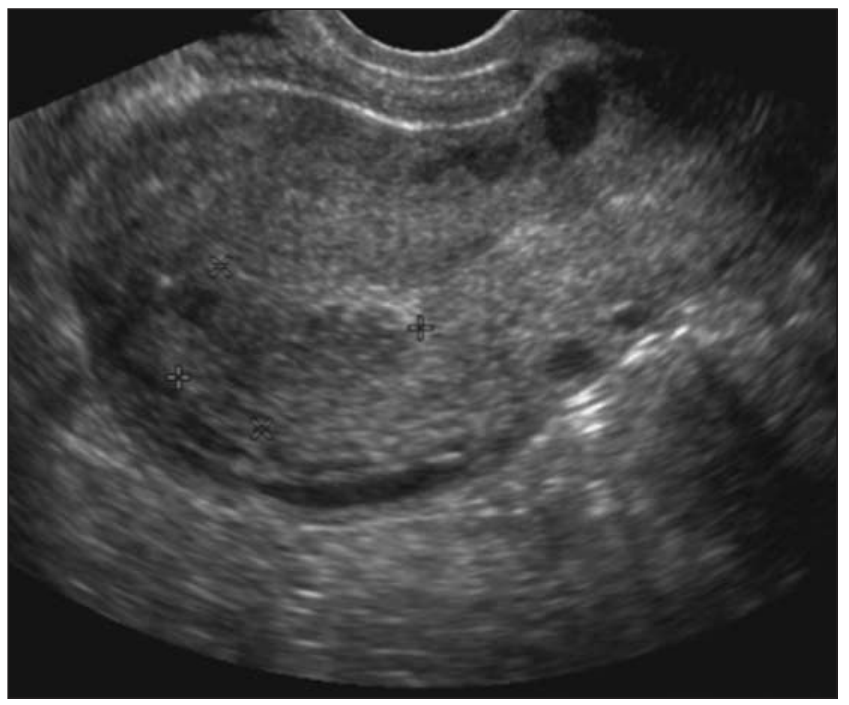

A

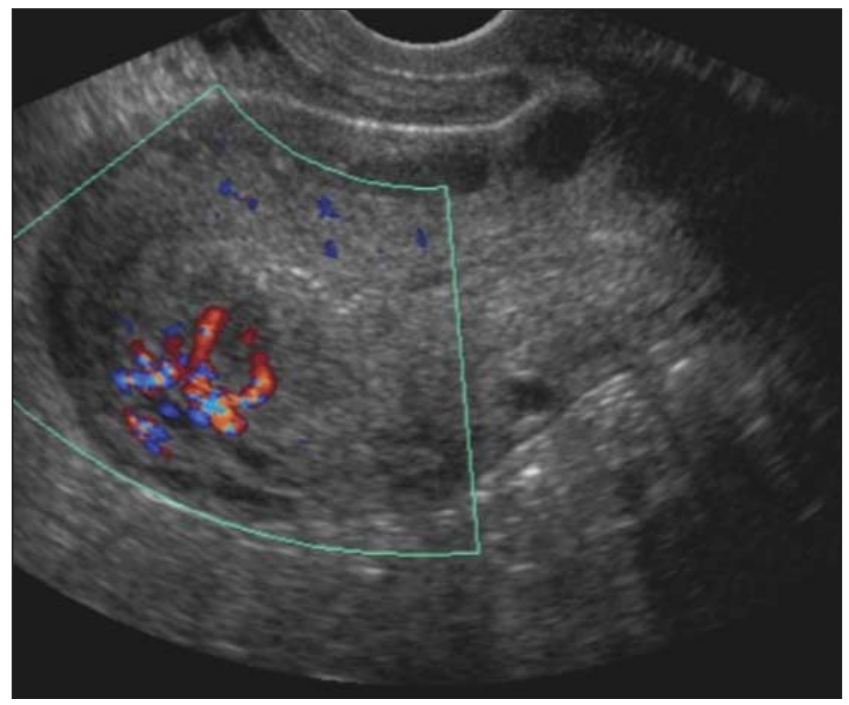

B

Figure 3. Transvaginal sonography without (A) and with (B) color Doppler imaging shows findings characterized as a vascular uterine mass, which was thought to be exclusively endometrial according to one radiologist and as having myometrial involvement according to the other radiologist. The final pathologic diagnosis was RPOC.

diagnosing RPOC on sonography is further supported by a retrospective study of 156 postpartum and postabortion patients by Sadan et $\mathrm{al},{ }^{5}$ which found an overall false-positive rate for RPOC of $34 \%$. Our study emphasizes another challenge of diagnosing RPOC on sonography because of the variety of potential appearances that RPOC can have, including a myometrial location and hypervascularity.
Sonography can be used to evaluate color Doppler flow of uterine abnormalities but has not been found to be very reliable in helping distinguish RPOC. It has been reported that hypervascularity on color Doppler imaging can occur in a variety of conditions, including normal pregnancy, gestational trophoblastic disease, and RPOC. ${ }^{6}$ The previously mentioned study by Durfee et $\mathrm{al}^{3}$ found that color Doppler flow was

Figure 4. Transvaginal sonography without (A) and with (B) power Doppler imaging shows findings characterized as a vascular myometrial mass by both radiologists. Both radiologists included a uterine AVM in the differential diagnosis. The final pathologic diagnosis was RPOC.

A

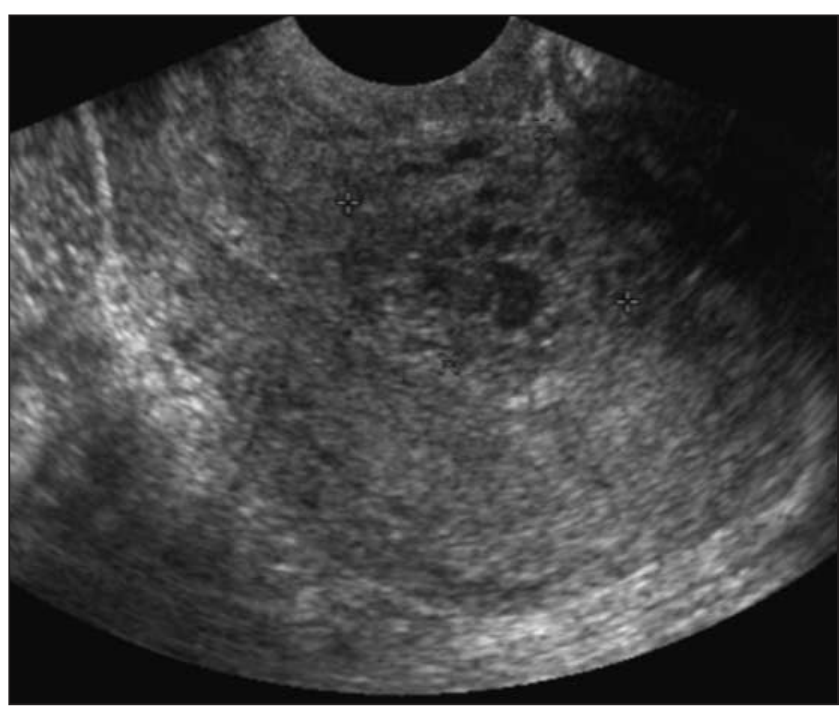

B

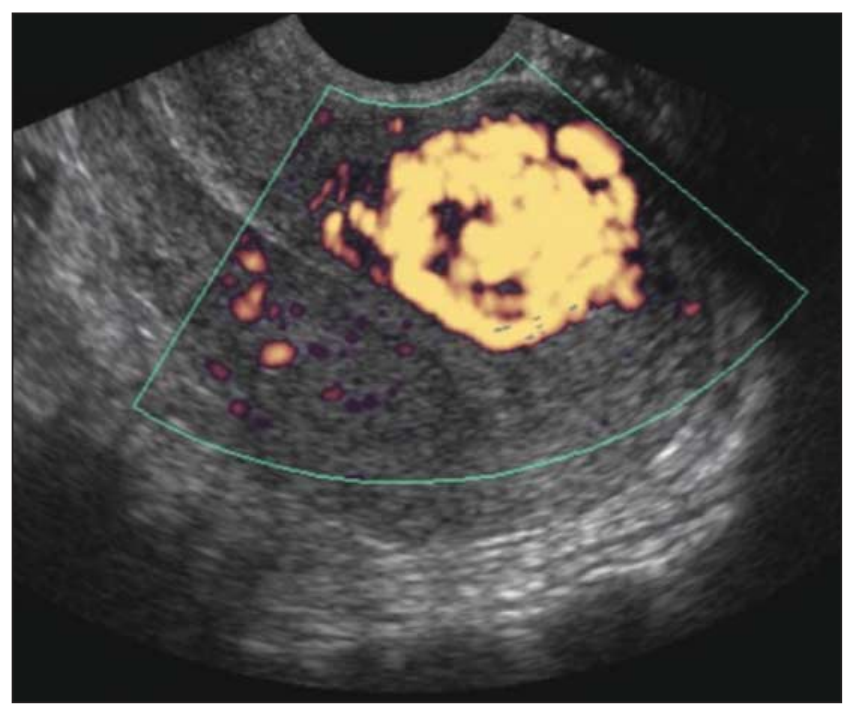


not helpful in distinguishing between the presence or absence of RPOC, even though vascular flow was seen somewhat more commonly in the presence of RPOC. Our study also supports the nonspecificity of color Doppler flow because some pathologically proven cases of RPOC were associated with a vascular abnormality, whereas others were not. Our findings also show just how exuberant color Doppler flow can be with RPOC, at times even mimicking a more vascular lesion such as a uterine AVM.

There are reported cases in the literature of RPOC mimicking a uterine AVM. One case report by Jain and Fogata ${ }^{7}$ involved a patient with a highly vascular lobulated mass in the endometrial cavity, which showed low resistance and a high peak systolic velocity on spectral Doppler imaging, suggestive of arteriovenous shunting. After spontaneous vaginal passage of tissue, the sonographic findings disappeared, with pathologic analysis of the tissue revealing RPOC. Another case report by Kido et $\mathrm{al}^{8}$ involved a patient with a poorly defined area of myometrial inhomogeneity in the anterior uterine wall that showed intense color Doppler signals with aliasing and flow reversals throughout the anterior uterine wall. The patient eventually underwent a hysterectomy, with a final pathology report revealing RPOC. Our study also emphasizes how hypervascularity of RPOC can lead to difficulty in imaging diagnosis, particularly when the area of hypervascularity involves the myometrium.

Myometrial abnormalities on sonography have previously been associated with uterine AVMs. Torres et $\mathrm{al}^{9}$ first described the gray scale findings of uterine AVM in 1979 as "multiple structures with a serpentine contour within the myometrium." Another more recent study of 21 women with symptomatic uterine AVMs by O'Brien et $\mathrm{al}^{10}$ found that the most common gray scale finding of a uterine AVM was myometrial heterogeneity, although the authors also emphasized that the gray scale sonographic findings of a uterine AVM were nonspecific. Our findings further confirm the nonspecific nature of myometrial uterine abnormalities on sonography because none of the cases with myometrial findings turned out to be uterine AVMs.
Uterine AVMs can be divided into congenital and acquired subtypes. ${ }^{11}$ Acquired uterine AVMs are usually traumatic and may be related to dilation and curettage (D\&C), therapeutic abortion, uterine surgery, or direct uterine trauma. ${ }^{12}$ Although rare, it is important to recognize uterine AVMs because treatment with D\&C could potentially cause serious bleeding. However, there is also a recognized potential to overdiagnose uterine AVMs in the postpartum and postabortion periods. ${ }^{13}$ Many socalled uterine AVMs diagnosed in the early postpartum or postabortion period spontaneously resolve on follow-up imaging. ${ }^{14}$ This issue has clinical importance because if curettage is not performed for fear of heavy bleeding related to a possible uterine AVM, the patient may undergo preventable blood loss due to the presence of RPOC. It may be reasonable to consider proceeding with $\mathrm{D} \& \mathrm{C}$ with angiographic backup available if there is a high clinical suspicion for RPOC in a patient with a vascular uterine mass.

Our study did have some recognized limitations, including the retrospective nature and relatively small sample size. Another limitation was that spectral Doppler findings were not evaluated. Spectral Doppler sonography was not a standard part of the imaging protocol during the study period. It is currently included in our imaging protocol, with the pulse repetition frequency optimized for high flow. Additionally, there were no pathologically proven uterine AVMs in our study, which likely reflects the rarity of this diagnosis. The true incidence of uterine AVMs is unknown.

In conclusion, despite high interobserver agreement in characterizing postpartum and postabortion uterine abnormalities on sonography, readers still include uterine AVMs in the differential diagnosis of vascular uterine masses that are ultimately proven to be RPOC. A myometrial location is a particularly misleading imaging feature of RPOC. In these instances, correlation with $\beta$-human chorionic gonadotropin measurements and noninvasive imaging such as follow-up pelvic sonography or magnetic resonance imaging may be helpful in the treatment of these challenging patients. 


\section{References}

1. Shen $O$, Rabinowitz $R$, Eisenberg VH, Samueloff $A$. Transabdominal sonography before uterine exploration as a predictor of retained placental fragments. J Ultrasound Med 2003; 22:561-564.

2. Huang MW, Muradali D, Thurston WA, Burns PN, Wilson SR. Uterine arteriovenous malformations: gray-scale and Doppler US features with MR imaging correlation. Radiology 1998; 206:115-123.

3. Durfee $S$, Frates M, Luong A, Benson C. The sonographic and color Doppler features of retained products of conception. J Ultrasound Med 2005; 24:1181-1186.

4. Hertzberg BS, Bowie JD. Ultrasound of the postpartum uterus: prediction of retained placental tissue. J Ultrasound Med 1991; 10:451-456.

5. Sadan O, Golan A, Girtler O, et al. Role of sonography in the diagnosis of retained products of conception. J Ultrasound Med 2004; 23:371-374.

6. Laing FC, Frates MC. Ultrasound evaluation during the first trimester of pregnancy. In: Callen PW (ed). Ultrasonography in Obstetrics and Gynecology. Philadelphia, PA: WB Saunders Co; 2000:127-128.

7. Jain K, Fogata M. Retained products of conception mimicking a large endometrial AVM: complete resolution following spontaneous abortion. J Clin Ultrasound 2007; 35:42-47.

8. Kido A, Togashi K, Koyama T, et al. Retained products of conception masquerading as acquired arteriovenous malformation. J Comput Assist Tomogr 2003; 27:88-92.

9. Torres WE, Sones PJ Jr, Thames FM. Ultrasound appearance of a pelvic arteriovenous malformation. J Clin Ultrasound 1979; 7:383-385.

10. O'Brien P, Neyastani A, Buckely AR, Chang SD, Legiehn GM. Uterine arteriovenous malformations: from diagnosis to treatment. J Ultrasound Med 2006; 25:1387-1392.

11. Vogelzang RL, Nemcek AA Jr, Skrtic Z, Gorrell J, Jurain JR. Uterine arteriovenous malformations: primary treatment with therapeutic embolization. J Vasc Interv Radiol 1991; 2:517-522.

12. Fleming $\mathrm{H}$, Ostor $A \mathrm{G}$, Pickel $\mathrm{H}$, Fortune DW. Arteriovenous malformations of the uterus. Obstet Gynecol 1989; 73: 209-213.

13. Mungen E. Vascular abnormalities of the uterus: have we recently overdiagnosed them? Ultrasound Obstet Gynecol 2003; 21:529-531.

14. Brown DL. Pelvic ultrasound in the postabortion and postpartum patient. Ultrasound Q 2005; 21:27-37. 